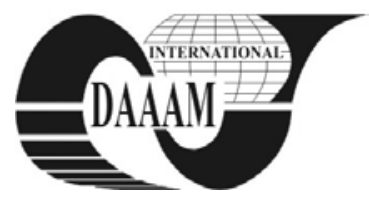

\title{
THE MOBILE MONITORING AND CONTROLLING OF REAL SYSTEMS VIA THE UMTS
}

\author{
MATYSEK, M[iroslav]; ADAMEK, M[ilan]; NEUMANN, P[etr] \& KARAFIAT, T[omas]
}

\begin{abstract}
The goal of this work was to design and realize a remote real system monitoring and controlling with the help of a mobile phone in the client/server structure. The client segment running in a UMTS mobile phone was realized in the JME environment. Internet data transfer is supported by TCP/IP protocol and mobile data transfer is supported by HSDPA / HSUPA service. The server segment was created in JSE. A simple desktop application controlled via the system concerned is a part of the server segment running on a PC

Key words: mobile monitoring, mobile control, real systems, UMTS, java
\end{abstract}

\section{INTRODUCTION}

There are not many professional systems for the remote monitoring and controlling of simple real systems via UMTS (Universal Mobile Telecommunication System) HSDPA / HSUPA (High Speed Downlink Packet Access) / (High Speed Uplink Packet Access) data services in Czech Republic.

The mobile monitoring and controlling system has been exclusively designed for simple real systems with slower events occurrence because of limited response time with mobile HSDPA/HSUPA data transfer.

This work was inspired with some similarly working mobile phone applications. These applications are programmed in Java language.

\section{THE SOLUTION PROPOSAL}

The client/server architecture has been chosen for a real system controlling and monitoring via mobile phone. The client application has been realized in JME (Java Micro Edition) again because of its massive support by mobile phone producers (Mahmoud, 2002). The applications are easily transferable among mobile phones independently from brand or platform (Topley, 2004).

The following main requirements are imposed on the client application:

- It should connect to the server exclusively when needed and send minimum data for sake of low costs

- It sends commands for system setting according to the user requirements

- It receives information on real system latest state, and it displays it on display.

The following main requirements are imposed on the client application:

- It coordinates the communication with client

- It receives client commands for the system setting

- It dispatches the information on system current state to the client

- It controls the system according to the client commands

- It reads the latest information from the system.

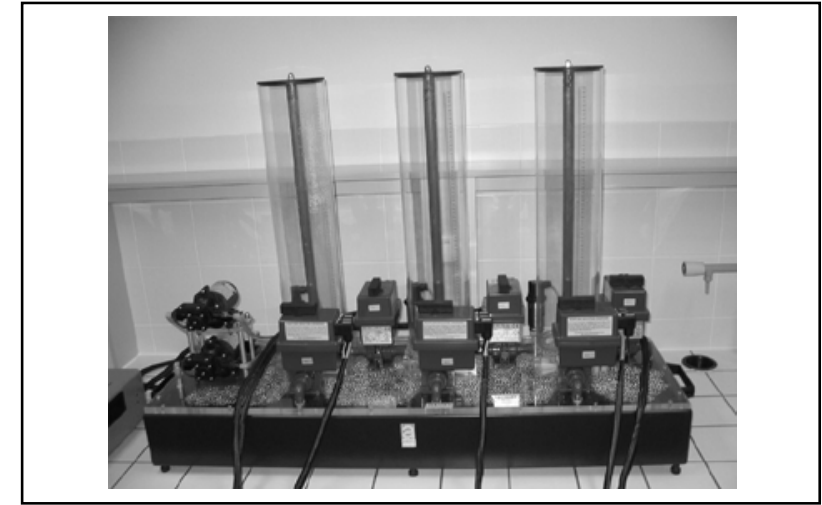

Fig. 1. Real system Amira DTS200

\section{MOBILE PHONE}

There was the mobile phone Nokia E51 employed for the system control and monitoring. That model was applied in spite of its 3 year old design because it supports all important Java and UMTS HSDPA specifications and because of its convenient dimensions unlike some current models (Kaaranen \& coll., 2005).

\section{THE REAL SYSTEM}

That real system used for our work results confirmation had been chosen from a couple of reasons. One of those reasons is the results vivid presentation and the other is that the events happening are "slow enough" to be controlled via mobile phone and UMTS network.

The water system DTS200 (Fig.1.) by Amira company contents three tanks N1, N2, and N3 which are interconnected with valves.

Each tank has an extra valve for draining the tank liquid into a container with exception of N3 that has two drain valves. The pump P1 supplies liquid to the tank N1 and the pump P2 supplies the tank N3.

\section{NETBEANS}

The development environment NetBeans IDE (Integrated Development Environment) version 6.8 was applied during both the mobile phone application and the PC server application development (Buchalcevova \& Pitka, 2007). The mobile phone application was tested in the environment inbuilt emulator (Bock, 2010).

\section{JAVA}

\subsection{Java Development Kit}

The JDK (Java Development Kit) represents the set of basic tools for Java platform application development so that it has to 
be installed for all Java applications development, including JSE (Java Standard Edition) and JME.

\subsection{Java Platform Micro Edition Software Development Kit} We need JME SDK (it replaces popular Java Wireless Toolkit) besides JDK for the mobile applications in Java.

\subsection{Java Runtime Environment}

Providing we plan exclusively to run Java applications, the JRE (Java Runtime Environment) installation is sufficient.

\section{MATLAB}

The adopted real system is commonly controlled from MATLAB environment (version 2007a) with Real Time Toolbox support. The necessary drivers are booted easily, and the control is possible from the command line or via the Simulink graphic environment.

\section{MATLAB CONTROL}

Matlabcontrol is a Java API that facilitates the control and Java applications transaction with local or remote MATLAB sessions.

The Matlabcontrol interface was used for communication with the real system as a remote session in this project. It means that the Java application (the simple server running on the controlling PC in our case) creates a MATLAB session after start and it asks for technological card driver boot. The device linked to the technological card is to be controlled from Java application through MATLAB commands then.

\section{CLIENT APPLICATION}

The controlling and monitoring systém client application was created as a project in JME NetBeans, CLDC 1.1 configuration and MIDP 2.0 profile. Unlike the server application, the client source code covers the user graphic interface and operation. The graphic interface has been created with NetBeans FlowDesigner RAD tool.

\subsection{Start screen}

It is a splashScreen object and it is displayed during application start-up procedure.

\subsection{Main menu}

The list type screen contents main menu items Monitoring, Controls, Application setup, About system and About application (Fig. 2.).

\subsection{Forms}

- Monitoring submenu - displays the information about system devices what means tank water level in \%, state of pumps and valves (Fig. 2.)

- Controls submenu - offers the possibility to change the system devices state what means to switch on or off pumps, open or close valves (Fig. 3.)

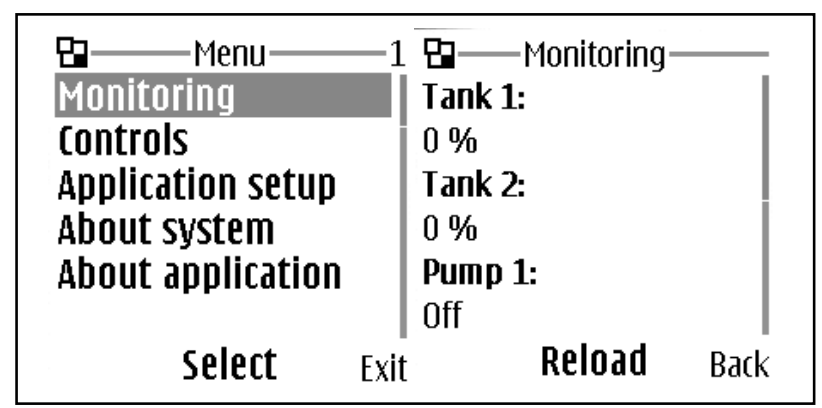

Fig. 2. Main menu and Monitoring on mobile phone

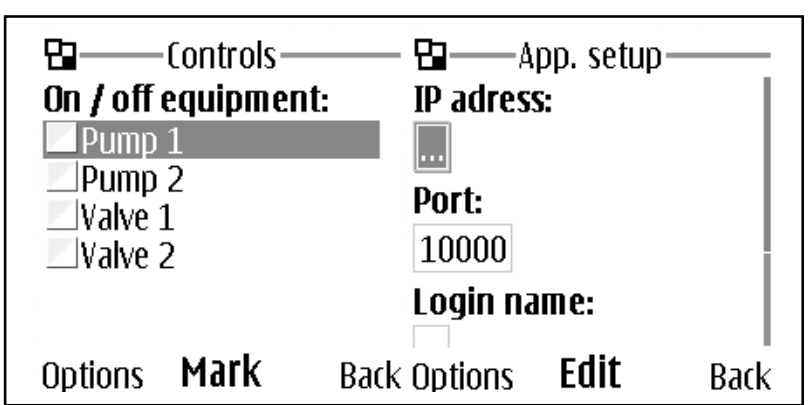

Fig. 3. Controls and Application setup on mobile phone

- Application setup submenu - configuration data insertion: IP address, server port, server login name and password (Fig. 3.)

- About system submenu describes briefly the real system to be controlled

- About application submenu lists the application information.

\section{SERVER APPLICATION}

The control and monitoring system application was created as a project in JSE NetBeans environment.

The server is to be set before the start of first communication with the START button. We should insert values for port (port for the server application), client login name and password. After communication start, the server runs and waits for a client. There are messages about communication state a information and would-be errors displayed in the text information window.

\subsection{Results applicability}

There is basically not necessary to install the application. The JAR (Java ARchive) file execute is enough. We can run the application on any PC with JRE and MATLAB installed. JVM (Java Virtual Machine) must not be firewall blocked if we need the network functions to be exploited, and application PC has to be connected to the internet via public IP address.

\section{CONCLUSION}

The costs for remote controlling and monitoring system are very low thanks to UMTS HSDPA data transfer and small data volumes - about $0.8 \mathrm{kB}$ per one monitoring and control.

We would like to implement developed system on the central heating of houses.

\section{ACKNOWLEDGEMENTS}

This work was supported by the Ministry of Education of the Czech Republic in the range of research projects No. MSM 7088352102 .

\section{REFERENCES}

Bock, H. (2010). Platform NetBeans: Detailed programming guide, Computer Press, ISBN 978-80-251-3116-9, Brno

Buchalcevova, A. \& Pitka, L. (2007). NetBeans: Development environment, Oeconomica, ISBN 978-80-245-1206-8, Praha

Kaaranen, H. \& coll. (2005). UMTS Networks: Architecture, Mobility and Services, Wiley, ISBN 978-0470011034, Oxford

Mahmoud, Q. (2002). Learn Java 2 Micro Edition, Grada Publishing, ISBN 80-247-0444-7, Praha

Topley, K. (2004). J2ME in epitome: Reference manual, Grada Publishing, ISBN 80-247-0426-9, Praha 